\title{
Efficient Signcryption with Key Privacy from Gap Diffie-Hellman Groups
}

\author{
Benoît Libert* and Jean-Jacques Quisquater \\ UCL Crypto Group \\ Place du Levant, 3. B-1348 Louvain-La-Neuve. Belgium \\ \{libert,jjq\}@dice.ucl.ac.be - http://www.uclcrypto.org/
}

\begin{abstract}
This paper proposes a new public key authenticated encryption (signcryption) scheme based on the Diffie-Hellman problem in Gap Diffie-Hellman groups. This scheme is built on the scheme proposed by Boneh, Lynn and Shacham in 2001 to produce short signatures. The idea is to introduce some randomness into this signature to increase its level of security in the random oracle model and to re-use that randomness to perform encryption. This results in a signcryption protocol that is more efficient than any combination of that signature with an El Gamal like encryption scheme. The new scheme is also shown to satisfy really strong security notions and its strong unforgeability is tightly related to the Diffie-Hellman assumption in Gap Diffie-Hellman groups.
\end{abstract}

Keywords: signcryption, Gap Diffie-Hellman groups, provable security

\section{Introduction}

The concept of public key signcryption schemes was proposed by Zheng in 1997 (29]). The purpose of this kind of primitive is to perform encryption and signature in a single logical step in order to obtain confidentiality, integrity, authentication and non-repudiation more efficiently than the sign-then-encrypt approach. The drawback of this latter solution is to expand the final ciphertext size (this could be impractical for low bandwidth networks) and increase the sender and receiver's computing time. Several efficient signcryption schemes have been proposed since 1997. The original scheme proposed in [29] was based on the discrete logarithm problem but no security proof was given. Zheng's original construction was only proven secure in 2002 ([3] ) by Baek et al. who described a formal security model in a multi-user setting. In 2000, Steinfeld and Zheng ([27]) proposed another scheme for which the unforgeability of ciphertexts relies on the intractability of the factoring problem but they provided no proof of chosen ciphertext security.

The drawback of the previously cited solutions is that they do not offer easy non-repudiation of ciphertexts: a recipient cannot prove to a third party that some plaintext was actually signcrypted by the sender. Bao and Deng ([5]) proposed a method to add universal verifiability to Zheng's cryptosystem but their

* This author was supported by the DGTRE's First Europe Project.

(C) International Association for Cryptologic Research 2004 
scheme was shown ([26]) to leak some information about the plaintext as other schemes like 28. The latter schemes can easily be modified to fix their problem but no strong guarantee of unforgeability can be obtained for them since the unforgeability of ciphertexts relies on the forking lemma (24, 25]]) which does not provide tight security reductions (see 16 for details). In the discrete logarithm setting, another scheme was shown in [26] to be chosen ciphertext secure under the Gap Diffie-Hellman assumption but it was built on a modified version of the DSA signature scheme which is not provably secure currently. As a consequence, no proof of unforgeability could be found for that scheme. An RSA-based scheme was described by Malone-Lee and Mao (20]) who provided proofs for both unforgeability under chosen-message attacks and chosen ciphertext security. Unfortunately, they only considered a security in a singleuser setting rather than the more realistic multi-user setting. Furthermore, the security of that scheme is only loosely related to the RSA assumption. However, none of these schemes is provably secure against insider attacks: in some of them, an attacker learning some user's private key can recover all messages previously signcrypted by that user.

In 2002, An et al. (1]) presented an approach consisting in performing signature and encryption in parallel: a plaintext is first transformed into a pair $(c, d)$ made of a commitment $c$ and a de-commitment $d$ in such a way that $c$ reveals no information about $m$ while the pair $(c, d)$ allows recovering $m$. Once he completed the transformation, the signer can jointly encrypt $c$ and sign $d$ in parallel using appropriate encryption and signature schemes. The de-signcryption operation is then achieved by the recipient in a parallel fashion: the signature on $d$ is verified while $c$ is decrypted and the pair $(c, d)$ is then used to recover the plaintext. This method decreases the computation time to signcrypt a message to the maximum of the times required by the underlying encryption and signature processes but the commitment step unfortunately involves some computation overhead. To improve this parallel approach, Pieprzyk and Pointcheval ([22]) proposed to use a $(2,2)$-Shamir secret sharing as an efficient commitment scheme: a plaintext is first splitted into two shares $s_{1}, s_{2}$ which do not individually reveal any information on $m . s_{1}$ is used as a commitment and encrypted while $s_{2}$ is signed as a de-commitment. The authors of 22 also gave a construction allowing them to integrate any one-way encryption system (such as the basic RSA) with a weakly secure signature (non-universally forgeable signatures in fact) into a chosen ciphertext secure and existentially unforgeable signcryption scheme.

Dodis et al. ([1] ) recently proposed another technique to perform parallel signcryption. Their method consists in a Feistel probabilistic two-paddings (called PSEP for short) which can be viewed as a generalization of other existing probabilistic paddings (OAEP, OAEP +, PSS-R,etc.) and involve a particular kind of commitment schemes. The authors of [11] showed that their construction also allows optimal exact security, flexible key management, compatibility with PKCS standards and has other interesting properties. They also claim that their scheme outperforms all existing signcryption solutions. We do not agree with that point since their method, like all other parallel signcryption proposi- 
tions, has a significant drawback: the recipient of a message is required to know from whom a ciphertext emanates before beginning to verify the signature in parallel with the decryption operation. A trivial solution to this problem would be to append a tag containing the sender's identity to the ciphertext but this would prevent the scheme from satisfying the notion of ciphertext anonymity formalized by Boyen in 10 (intuitively, this notion expresses the inability for someone observing a ciphertext to determine who the sender is nor to whom it is intended) that can be a desirable feature in many applications (see 10 for examples). Furthermore, by the same arguments as those in [6], one can easily notice that the probabilistic padding described in 11 does not allow the key privacy property to be achieved when instantiated with trapdoor permutations such as RSA, Rabin or Paillier: in these cases, given a ciphertext and a set of public keys, it is possible to determine under which key the message was encrypted. An anonymous trapdoor permutation or a repeated variant of the padding PSEP (as the solutions proposed in [6]) could be used to solve this problem but this would decrease the scheme's efficiency.

In this paper, we propose a new discrete logarithm based signcryption scheme which satisfies strong security notions: chosen ciphertext security against insider attacks (except the hybrid composition proposed in [17] and the identity based scheme described in [10, no discrete logarithm based authenticated encryption method was formally proven secure in such a model before), strong unforgeability against chosen-message attacks, ciphertext anonymity in the sense of [10] (this is an extension of the notion of key privacy proposed in [6] to the signcryption case). We also prove that it satisfies a new security notion that is related to the one of ciphertext anonymity and that we call 'key invisibility'. We show that the scheme's strong unforgeability is really tightly related to the hardness of the Diffie-Hellman problem unlike the scheme proposed in 10] whose proof of unforgeability relies on Pointcheval and Stern's forking lemma and thus only provides a loose reduction to a computational problem. In fact, except the hybrid construction of [17] (whose semantic security is based on the stronger hash oracle Diffie-Hellman assumption) our scheme appears to be the first discrete logarithm based signcryption protocol whose (strong) unforgeabililty is proven to be tightly related to the Diffie-Hellman problem. About the semantic security of the scheme, we give heuristic arguments showing that it is more tightly related to the Diffie-Hellman problem than expressed by the bounds at first sight. Unlike [1], 11] and [22, our protocol is sequential but it is efficient and does not require the recipient of a message to know who is the sender before starting the de-signcryption process. Our scheme borrows a construction due to Boyen (10]) and makes extensive use of the properties of some bilinear maps over the so-called Gap Diffie-Hellman groups (in fact, the structure of these groups is also exploited in our security proofs). Before describing our scheme, we first recall the properties of these maps in section 2 . The section 3 formally describes the security notions that our scheme, depicted in section 4 , is shown to satisfy in the security analysis presented in section 5 . 


\section{Preliminaries}

\subsection{Overview of Pairings}

Let $k$ be a security parameter and $q$ be a $k$-bit prime number. Let us consider groups $\mathbb{G}_{1}$ and $\mathbb{G}_{2}$ of the same prime order $q$. For our purposes, we need a bilinear map $\hat{e}: \mathbb{G}_{1} \times \mathbb{G}_{1} \rightarrow \mathbb{G}_{2}$ satisfying the following properties:

1. Bilinearity: $\forall P, Q \in \mathbb{G}_{1}, \forall a, b \in \mathbb{Z}_{q}^{*}$, we have $\hat{e}(a P, b Q)=\hat{e}(P, Q)^{a b}$.

2. Non-degeneracy: for any $P \in \mathbb{G}_{1}, \hat{e}(P, Q)=1$ for all $Q \in \mathbb{G}_{1}$ iff $P=\mathcal{O}$.

3. Computability: an efficient algorithm allows computing $\hat{e}(P, Q) \forall P, Q \in \mathbb{G}_{1}$.

The modified Weil pairing ([8] ) and the Tate pairing are admissible maps of this kind. The group $\mathbb{G}_{1}$ is a suitable cyclic elliptic curve subgroup while $\mathbb{G}_{2}$ is a cyclic subgroup of the multiplicative group associated to a finite field. We now recall some problems that provided underlying assumptions for many previously proposed pairing based cryptosystems. These problems are formalized according to the elliptic curve additive notation.

Definition 1. Given groups $\mathbb{G}_{1}$ and $\mathbb{G}_{2}$ of prime order $q$, a bilinear map $\hat{e}$ : $\mathbb{G}_{1} \times \mathbb{G}_{1} \rightarrow \mathbb{G}_{2}$ and a generator $P$ of $\mathbb{G}_{1}$,

- The Computational Diffie-Hellman problem (CDH) in $\mathbb{G}_{1}$ is, given $\langle P, a P, b P\rangle$ for unknown $a, b \in \mathbb{Z}_{q}$, to compute $a b P \in \mathbb{G}_{1}$.

- The Decisional Diffie-Hellman problem (DDH) is, given $\langle P, a P, b P, c P\rangle$ for unknown $a, b, c \in \mathbb{Z}_{q}$, to decide whether $a b \equiv c(\bmod q)$ or not. Tuples of the form $\langle P, a P, b P, c P\rangle$ for which the latter condition holds are called "Diffie-Hellman tuples".

- The Gap Diffie-Hellman problem (GDH) is to solve a given instance $\langle P, a P, b P\rangle$ of the CDH problem with the help of a DDH oracle that is able to decide whether a tuple $\left\langle P, a^{\prime} P, b^{\prime} P, c^{\prime} P\right\rangle$ is such that $c^{\prime} \equiv a^{\prime} b^{\prime}(\bmod q)$.

As shown in 18, a pairing can implement a DDH oracle. Indeed, in a group $\mathbb{G}_{1}$ for which pairings are efficiently computable, to determine whether a tuple $\langle P, a P, b P, c P\rangle$ is a valid Diffie-Hellman tuple or not, it suffices to check if $\hat{e}(P, c P)=\hat{e}(a P, b P)$. This kind of group, where the DDH problem is easy while the $\mathrm{CDH}$ one is still believed to be hard, is called Gap Diffie-Hellman groups in the literature $([18,[21])$.

\section{Security Notions for Signcryption Schemes}

We first recall the two usual security notions: the security against chosen ciphertext attacks which is also called semantic security and the unforgeability against chosen-message attacks. We then consider other security notions that were proposed by Boyen ([10]) in 2003. In the notion of chosen ciphertext security, we consider a multi-user security model as already done in [1], [3], 11], [22] and [10] to allow the adversary to query the de-signcryption oracle on ciphertexts created 
with other private keys than the attacked one. We also consider the security against insider attacks by allowing the attacker to choose to be challenged on a signcrypted text created by a corrupted user (i.e. a user whose private key is known to the attacker). Indeed, for confidentiality purposes, we require the owner of a private key to be unable to find any information on a ciphertext created with that particular key without knowing which randomness was used to produce that ciphertext. As already considered in 11, 10, [11 and 22, this also allows us showing that an attacker stealing a private key does not threaten the confidentiality of messages previously signcrypted using that private key.

Definition 2. We say that a signcryption scheme is semantically secure against chosen ciphertext attacks (we call this security notion SC-IND-CCA) if no probabilistic polynomial time (PPT) adversary has a non-negligible advantage in the following game:

1. The challenger runs the key generation algorithm Keygen to generate a private/public key pair $\left(s k_{U}, p k_{U}\right) . s k_{U}$ is kept secret while $p k_{U}$ is given to the adversary $\mathcal{A}$.

2. $\mathcal{A}$ performs a first series of queries in a first stage. These queries can be of the following kinds:

- Signcryption queries: $\mathcal{A}$ produces a message $m \in \mathcal{M}$ and an arbitrary public key $p k_{R}$ (that public key may differ from $p k_{U}$ ) and requires the result Signcrypt $\left(m, s k_{U}, p k_{R}\right)$ of the signcryption oracle.

- De-signcryption queries: $\mathcal{A}$ produces a ciphertext $\sigma$ and requires the result of the operation De-signcryt $\left(\sigma, s k_{U}\right)$. This result is made of a signed plaintext and a sender's public key if the obtained signed-plaintext is valid for the recovered sender's public key. Otherwise (that is if the obtained plaintext-signature pair is not valid for the obtained public key when performing the de-signcryption operation with the private key $\left.s k_{U}\right)$, the $\perp$ symbol is returned as a result.

These queries can be asked adaptively: each query may depend on the answers to previous ones.

3. $\mathcal{A}$ produces two plaintexts $m_{0}, m_{1} \in \mathcal{M}$ of equal size and an arbitrary private key sk $k_{S}$. The challenger then flips a coin $b \leftarrow_{R}\{0,1\}$ to compute a signcryption $\sigma=S i g n c r y p t\left(m_{b}, s k_{S}, p k_{U}\right)$ of $m_{b}$ with the sender's private key $s k_{S}$ under the attacked receiver's public key $p k_{U} . \sigma$ is sent to $\mathcal{A}$ as a challenge.

4. The adversary performs new queries as in the first stage. Now, it may not ask the de-signcryption of the challenge $\sigma$ with the private key $s k_{U}$ of the attacked receiver.

5. At the end of the game, $\mathcal{A}$ outputs a bit $b^{\prime}$ and wins if $b^{\prime}=b$.

$\mathcal{A}$ 's advantage is defined to be $\operatorname{Adv} v^{i n d-c c a}(\mathcal{A}):=2 \operatorname{Pr}\left[b^{\prime}=b\right]-1$.

In the notion of unforgeability captured by the formal definition below, as in many other previous works $([1],[3,[10],[11],[17,, 22]$, etc. $)$, we allow a forger attempting to forge a ciphertext on behalf of the attacked user $U$ to know the receiver's private key. In fact, the attacker has to come with the intended receiver's private key $s k_{R}$ as a part of the forgery. The motivation is to prove that 
no attacker can forge a ciphertext intended to any receiver on behalf of a given sender. In particular, no dishonest user can produce a ciphertext intended to himself and try to convince a third party that it emanates from a honest user.

Definition 3. We say that a signcryption scheme is strongly existentially unforgeable against chosen-message attacks (SC-SUF-CMA) if no PPT adversary has a non-negligible advantage in the following game:

1. The challenger generates a key pair $\left(s k_{U}, p k_{U}\right)$ and $p k_{U}$ is given to the forger $\mathcal{F}$.

2. The forger $\mathcal{F}$ queries the oracles $\operatorname{Signcryp}_{\text {sk }_{U}}(.,$.$) and De-signcrypt$ sk $_{U}($. exactly as in the previous definition. Again, these queries can also be produced adaptively.

3. At the end of the game, $\mathcal{F}$ produces a ciphertext $\sigma$ and a key pair $\left(s k_{R}, p k_{R}\right)$ and wins the game if the result of the operation De-signcrypt $\left(\sigma, s k_{R}\right)$ is a tuple $\left(m, s, p k_{U}\right)$ such that $(m, s)$ is a valid signature for the public key $p k_{U}$ such that $\sigma$ was not the output of a signcryption query Signcrypt $\left(m, s k_{U}, p k_{R}\right)$ made during the game.

Recall that, in the corresponding notion of conventional (i.e. non-strong) unforgeability for signcryption schemes, the attacker cannot win if the outputted ciphertext was the result of any signcryption query. In our context, as in [1], 17, 11, and many other works, the forger is allowed to have obtained the forged ciphertext as the result of a signcryption query for a different receiver's public key than the one corresponding to the claimed forgery. The only constraint is that, for the message $m$ obtained by de-signcryption of the alleged forgery with the chosen private key $s k_{R}$, the outputted ciphertext $\sigma$ was not obtained as the result of a Signcrypt $\left(m, s k_{U}, p k_{R}\right)$ query.

In [10, Boyen also proposed additional security notions for signcryption schemes. One of the most important ones was the notion of ciphertext anonymity that can be viewed as an extension to authenticated encryption schemes of the notion of key privacy already considered by Bellare et al in [6]. Intuitively, in the context of public key encryption, a scheme is said to have the key privacy property if ciphertexts convey no information about the public key that was used to create them. In the signcryption setting, we say that the ciphertext anonymity (or key privacy) property is satisfied if ciphertexts contain no information about who created them nor about to whom they are intended. This notion is a transposition into the non-identity based setting of the one presented in [10]. It can be described like that.

Definition 4. A signcryption scheme is said to satisfy the ciphertext anonymity property (also called key privacy or key indistinguishability: we call this notion $S C$-INDK-CCA for short) if no PPT distinguisher has a non-negligible advantage in the following game:

1. The challenger generates two key pairs $\left(s k_{R, 0}, p k_{R, 0}\right)$ and $\left(s k_{R, 1}, p k_{R, 1}\right)$. $p k_{R, 0}$ and $p k_{R, 1}$ are given to the distinguisher $\mathcal{D}$. 
2. $\mathcal{D}$ adaptively performs queries Signcrypt $\left(m, s k_{R, c}, p k_{R}\right)$, for arbitrary recipient keys $p k_{R}$, and De-signcrypt $\left(\sigma, s k_{R, c}\right)$ for $c=0$ or $c=1$.

3. Once stage 2 is over, $\mathcal{D}$ outputs two private keys $s k_{S, 0}$ and $s k_{S, 1}$ and a plaintext $m \in \mathcal{M}$. The challenger then flips two coins $b, b^{\prime} \leftarrow_{R}\{0,1\}$ and computes a challenge ciphertext $\sigma=\operatorname{Signcrypt}\left(m, s k_{S, b}, p k_{R, b^{\prime}}\right)$ which is sent to $\mathcal{D}$.

4. $\mathcal{D}$ adaptively performs new queries as in stage 2 with the restriction that, this time, it is disallowed to ask the de-signcryption of the challenge $\sigma$ with the private keys $s k_{R, 0}$ or $s k_{R, 1}$.

5. At the end of the game, $\mathcal{D}$ outputs bits $d, d^{\prime}$ and wins if $\left(d, d^{\prime}\right)=\left(b, b^{\prime}\right)$. Its advantage is defined to be $A d v^{\text {indk-cca }}(D):=\operatorname{Pr}\left[\left(d, d^{\prime}\right)=\left(b, b^{\prime}\right)\right]-1 / 4$.

Again, this notion captures the security against insider attacks since the distinguisher is allowed to choose a set of two private keys among which the one used as sender's key to create the challenge ciphertext is picked by the challenger. The above definition can be viewed as a transposition to the non-identity based setting of the definition of ciphertext anonymity proposed by Boyen ([10]) as well as an extension of the definition of key privacy ([6]) to the authenticated encryption context. We introduce another notion called 'key invisibility' which is close to the concept (formalized by Galbraith and Mao in [14]) of invisibility for undeniable signatures. Intuitively, this notion expresses the impossibility to decide whether a given ciphertext was actually created using a given particular sender's private key and a given particular receiver's public key.

Definition 5. We say that a signcryption scheme satisfies the key invisibility (we denote this notion by SC-INVK-CCA for short) if no PPT distinguisher has a non-negligible advantage in the following game:

1. The challenger generates a private/public key pair $\left(s k_{U}, p k_{U}\right) \cdot p k_{U}$ is given to the distinguisher $\mathcal{D}$.

2. $\mathcal{D}$ adaptively performs queries Signcrypt $\left(m, s k_{U}, p k_{R}\right)$, for arbitrary recipient keys $p k_{R}$, and De-signcrypt $\left(\sigma, s k_{U}\right)$.

3. Once stage 2 is over, $\mathcal{D}$ outputs a private key $s k_{S}$ and a plaintext $m \in \mathcal{M}$. The challenger then flips a coins $b \leftarrow_{R}\{0,1\}$. If $b=0$, then the challenger returns an actual challenge ciphertext $\sigma=\operatorname{Signcrypt}\left(m, s k_{S}, p k_{U}\right)$ to $\mathcal{D}$. If $b=1$, then the challenger returns a random $\sigma$ uniformly taken from the ciphertext space $\mathcal{C}$.

4. $\mathcal{D}$ adaptively performs new queries as in stage 2 with the restriction that, this time, it cannot require the de-signcryption of the challenge $\sigma$ with the private keys sk $_{U}$.

5. At the end of the game, $\mathcal{D}$ outputs bits $d$ and wins if $d=b$. Its advantage is defined as $A d v^{i n v k-c c a}(D):=2 \operatorname{Pr}[d=b]-1$.

Again, we allow the distinguisher to choose which private key is used as a part of the challenge to take insider attacks into account.

Galbraith and Mao (14]) showed that anonymity and invisibility are essentially equivalent security notions for undeniable signatures. While one can prove 
in the same way that key privacy and key invisibility are also essentially equivalent for some particular encryption schemes, such an equivalence turns out to be unclear in the signcryption case. In fact, one cannot prove that a distinguisher against the key invisibility implies a distinguisher against the key privacy with the same advantage (because two random coins are used by the challenger in the definition of key privacy and a single one for key anonymity). However, we can prove that, for signcryption schemes satisfying some particular properties (that is, for a given message and a given sender's private key, the output of the signcryption algorithm must be uniformly distributed in the ciphertext space when the receiver's public key is random), we can prove that key invisibility implies key privacy. This will be showed in 19. In the next section we propose a scheme that satisfies both of them (in addition to the usual notions of semantic security and unforgeability) in the random oracle model.

\section{A Diffie-Hellman Based Signcryption Scheme with Key Privacy}

This section presents a signcryption scheme whose unforgeability under chosenmessage attacks is tightly related to the hardness of the computational DiffieHellman problem in Gap Diffie-Hellman groups. Our solution relies on the BLS signature (9]) whose security is enhanced by a random quantity $U$ which is used for encryption purposes but also acts as a random salt to provide a tighter security reduction to the Diffie-Hellman problem in $\mathbb{G}_{1}$ in the proof of unforgeability.

We assume that both the sender and the receiver agreed on public parameters: security parameters $k$ and $\ell$, cyclic groups $\mathbb{G}_{1}$ and $\mathbb{G}_{2}$ of prime order $q \geq 2^{k}$ such that $\ell$ is the number of bits required to represent elements of $\mathbb{G}_{1}$, a generator $P$ of $\mathbb{G}_{1}$ and a bilinear map $\hat{e}: \mathbb{G}_{1} \times \mathbb{G}_{1} \rightarrow \mathbb{G}_{2}$. They also agree on cryptographic hash functions $H_{1}:\{0,1\}^{n+2 \ell} \rightarrow \mathbb{G}_{1}, H_{2}: \mathbb{G}_{1}^{3} \rightarrow\{0,1\}^{\ell}$ and $H_{3}:\{0,1\}^{\ell} \rightarrow\{0,1\}^{n+\ell}$ where $n$ denotes the size of plaintexts (i.e. the message space is $\left.\mathcal{M}=\{0,1\}^{n}\right)$. The scheme consists of the following three algorithms (we recall that the symbol $\oplus$ denotes the bitwise exclusive OR).

Keygen: user $\mathrm{u}$ picks a random $x_{u} \leftarrow_{R} \mathbb{Z}_{q}$ and sets his public key to $Y_{u}=$ $x_{u} P \in \mathbb{G}_{1}$. His private key is $x_{u}$. We will denote the sender and the receiver respectively by $u=S$ and $u=R$ and their key pair by $\left(x_{S}, Y_{S}\right)$ and $\left(x_{R}, Y_{R}\right)$.

Signcrypt: to signcrypt a plaintext $m \in\{0,1\}^{n}$ intended to $R$, the sender $S$ uses the following procedure

1. Pick a random $r \leftarrow_{R} \mathbb{Z}_{q}$ and compute $U=r P \in \mathbb{G}_{1}$.

2. Compute $V=x_{S} H_{1}\left(m, U, Y_{R}\right) \in \mathbb{G}_{1}$.

3. Compute $W=V \oplus H_{2}\left(U, Y_{R}, r Y_{R}\right) \in\{0,1\}^{\ell}$ and then scramble the plaintext together with the sender's public key: $Z=\left(m \| Y_{S}\right) \oplus H_{3}(V) \in$ $\{0,1\}^{n+\ell}$.

The ciphertext is given by $\sigma=\langle U, W, Z\rangle \in \mathbb{G}_{1} \times\{0,1\}^{n+2 \ell}$. 
De-signcrypt: when receiving a ciphertext $\sigma=\langle U, W, Z\rangle$, the receiver $R$ has to perform the steps below:

1. Compute $V=W \oplus H_{2}\left(U, Y_{R}, x_{R} U\right) \in\{0,1\}^{\ell}$.

2. Compute $\left(m \| Y_{S}\right)=Z \oplus H_{3}(V) \in\{0,1\}^{n+\ell}$. Reject $\sigma$ if $Y_{S}$ is not a point on the curve on which $\mathbb{G}_{1}$ is defined.

3. Compute $H=H_{1}\left(m, U, Y_{R}\right) \in \mathbb{G}_{1}$ and then check if $\hat{e}\left(Y_{S}, H\right)=\hat{e}(P, V)$. If this condition does not hold, reject the ciphertext.

The consistency of the scheme is easy to verifiy. To prove to a third party that the sender $\mathrm{S}$ actually signed a plaintext $m$, the receiver just has to forward it $m$ and $\left(U, V, Y_{R}\right)$. The third party can then compute $H$ as in the step 3 of de-signcrypt and perform the signature verification as in the same step 3. We note that, in the signcryption algorithm, the recipient's public key must be hashed together with the pair $(m, U)$ in order to achieve the provable strong unforgeability.

As pointed out in [15, in some applications, it is interesting for the origin of a signcrypted text to be publicly verifiable (by firewalls for example). In some other applications, it is undesirable: indeed as explained in [10, in some cases, it is better for a signcrypted text not to convey any information about its sender nor about its intended receiver. This property, called anonymity of ciphertexts, is provided by the above scheme as shown in the next section.

From an efficiency point of view, we can easily verifiy that the above scheme is at least as efficient and more compact than any sequential composition of the BLS signature (9]) with any other Diffie-Hellman based chosen ciphertext secure encryption scheme $([2],[4,[12,13], 23]$, etc. $)$ : indeed only three scalar multiplications in $\mathbb{G}_{1}$ are required for the signcryption operation while 1 multiplication and 2 pairings must be performed in the de-signcryption process. A sequential combination of the BLS signature with the encryption scheme proposed in [2] would involve an additional multiplication at decryption. If we take $\ell \approx k \geq 160$ (by working with an appropriate elliptic curve), we see that ciphertexts are about 480 bits longer than plaintexts. Any combination of the BLS signature with a CCA-secure El Gamal type cryptosystem would result in longer final ciphertexts. With the same choice of parameters, a composition of the BLS signature with the lenth-saving El Gamal encryption scheme ([2]) would result in ciphertexts that would be 640 bits longer than plaintexts.

\section{$5 \quad$ Security Analysis}

In this section, we first show that an adversary against the SC-IND-CCA security of the scheme implies a PPT algorithm that can solve the Diffie-Hellman problem in $\mathbb{G}_{1}$ with high probability. This fact is formalized by the following theorem.

Theorem 1. In the random oracle model, if an adversary $\mathcal{A}$ has a non-negligible advantage $\epsilon$ against the SC-IND-CCA security of the above scheme when running in a time $t$ and performing $q_{S C}$ signcryption queries, $q_{D S C}$ de-signcryption queries and $q_{H_{i}}$ queries to oracles $H_{i}$ (for $i=1, \ldots, 4$ ), then there exists an 
algorithm $\mathcal{B}$ that can solve the $C D H$ problem in the group $\mathbb{G}_{1}$ with a probability $\epsilon^{\prime} \geq \epsilon-q_{H_{3}} q_{D S C} / 2^{2 k}$ in a time $t^{\prime}<t+\left(4 q_{D S C}+2 q_{H_{2}}\right) t_{e}$ where $t_{e}$ denotes the time required for one pairing evaluation.

Proof. The algorithm $\mathcal{B}$ runs $\mathcal{A}$ as a subroutine to solve the $\mathrm{CDH}$ problem in a polynomial time. Let $(a P, b P)$ be a random instance of the $\mathrm{CDH}$ problem in $\mathbb{G}_{1} . \mathcal{B}$ simulates $\mathcal{A}$ 's challenger in the game of definition 2 and starts it with $Y_{u}=b P \in \mathbb{G}_{1}$ as a challenge public key. $\mathcal{A}$ then adaptively performs queries as explained in the definition. To handle these queries, $\mathcal{B}$ maintains lists $L_{i}$ to keep track of the answers given to oracle queries on $H_{i}$ for $i=1,2,3$. Hash queries on $\mathrm{H}_{2}$ and $\mathrm{H}_{3}$ are treated in the usual way: $\mathcal{B}$ first checks in the corresponding list if the oracle's value was already defined at the queried point. If it was, $\mathcal{B}$ returns the defined value. Otherwise, it returns a random element from the appropriate range and updates the corresponding list. When a hash query $H_{1}\left(m, U, Y_{R}\right)$ is performed, $\mathcal{B}$ first looks if the value of $H_{1}$ was previously defined for the input $\left(m, U, Y_{R}\right)$. If it was, the previously defined value is returned. Otherwise, $\mathcal{B}$ picks a random $t \leftarrow_{R} \mathbb{Z}_{q}$, returns $t P \in \mathbb{G}_{1}$ as an answer and inserts the tuple $\left(m, U, Y_{R}, t\right)$ into $L_{1}$.

Now, let us see how signcryption and de-signcryption queries are dealt with:

- For a signcryption query on a plaintext $m$ with a recipient's public key $Y_{R}$ both chosen by the adversary $\mathcal{A}, \mathcal{B}$ first picks a random $r \leftarrow_{R} \mathbb{Z}_{q}$, computes $U=r P \in \mathbb{G}_{1}$ and checks if $L_{1}$ contains a tuple $\left(m, U, Y_{R}, t\right)$ indicating that $H_{1}\left(m, U, Y_{R}\right)$ was previously defined to be $t P$. If no such tuple is found, $\mathcal{B}$ picks a random $t \leftarrow_{R} \mathbb{Z}_{q}$ and puts the entry $\left(m, U, Y_{R}, t\right)$ into $L_{1} \cdot \mathcal{B}$ then computes $V=t Y_{u}=t(b P) \in \mathbb{G}_{1}$ for the random $t$ chosen or recovered from $L_{1}$. The rest follows as in the normal signcryption process: $\mathcal{B}$ computes $r Y_{R}$ (for the $Y_{R}$ specified by the adversary), runs the $H_{2}$ simulation process to obtain $h_{2}=H_{2}\left(U, Y_{R}, r Y_{R}\right)$, and then computes $W=V \oplus h_{2}$ and $Z=$ $\left(m \| Y_{u}\right) \oplus h_{3}$ where $h_{3}$ is obtained by simulation of the $H_{3}$ oracle on the input $V .(U, W, Z)$ is then returned as a signcryption of $m$ from the sender of public key $Y_{u}$ to the recipient of public key $Y_{R}$.

- For a de-signcryption query on a ciphertext $\langle U, W, Z\rangle$ and a sender's public key $Y_{S}$ both chosen by $\mathcal{A}, \mathcal{B}$ proceeds as follows: it scans the list $L_{2}$, looking for tuples $\left(U, Y_{u}, S_{i}, h_{2, i}\right)$ (with $\left.0 \leq i \leq q_{H_{2}}\right)$ such that $V_{i}=h_{2, i} \oplus W$ exists in an entry $\left(V_{i}, h_{3, i}\right)$ of $L_{3}$ and, for the corresponding elements $h_{3, i},\left(m_{i}, Y_{S, i}\right)=$ $h_{3, i} \oplus Z \in\{0,1\}^{n+\ell}$ is such that there exists an entry $\left(m_{i}, U, Y_{u}, h_{1, i}\right)$ in the list $L_{1}$. If no such tuples are found, the $\perp$ symbol is returned to $\mathcal{A}$. Otherwise, elements $\left(m_{i}, U, V_{i}, S_{i}, h_{1, i}\right)$ satisfying those conditions are kept for future examination. If one of them satisfies both $\hat{e}\left(P, S_{i}\right)=\hat{e}\left(U, Y_{u}\right)$ and $\hat{e}\left(Y_{S, i}, h_{1, i}\right)=\hat{e}\left(P, V_{i}\right)$, then $\left\langle m_{i},\left(U, V_{i}\right)\right\rangle$ is returned as a message-signature pair together with the sender's public key $Y_{S, i}$.

At the end of the first stage, $\mathcal{A}$ outputs two plaintexts $m_{0}$ and $m_{1}$ together with an arbitrary sender's private key $x_{S}$ and requires a challenge ciphertext built under the recipient's public key $Y_{u} . \mathcal{B}$ ignores $m_{0}$ and $m_{1}$ and randomly picks two binary strings $W \leftarrow_{R}\{0,1\}^{\ell}$ and $Z \leftarrow_{R}\{0,1\}^{n+\ell}$. A challenge ciphertext 
$\sigma=\langle U, W, Z\rangle=\langle a P, W, Z\rangle$ is then sent to $\mathcal{A}$ that then performs a second series of queries at a second stage. These queries are handled by $\mathcal{B}$ as those at the first stage. As done in many other papers in the literature, it is easy to show that $\mathcal{A}$ will not realize that $\sigma$ is not a valid signcryption for the sender's private key $x_{S}$ and the public key $Y_{u}$ unless it asks for the hash value $H_{2}(a P, b P, a b P)$. In that case, the solution of the Diffie-Hellman problem would be inserted in $L_{2}$ exactly at that moment and it does not matter if the simulation of $\mathcal{A}$ 's view is no longer perfect.

At the end of the game, $\mathcal{A}$ produces a result which is ignored by $\mathcal{B}$. The latter just looks into the list $L_{2}$ for tuples of the form $\left(a P, b P, D_{i},.\right)$. For each of them, $\mathcal{B}$ checks whether $\hat{e}\left(P, D_{i}\right)=\hat{e}(a P, b P)$ and, if this relation holds, stops and outputs $D_{i}$ as a solution of the $\mathrm{CDH}$ problem. If no tuple of this kind satisfies the latter equality, $\mathcal{B}$ stops and outputs "failure".

Now to assess $\mathcal{B}$ 's probability of success, let us denote by $\mathrm{AskH}_{2}$ the event that $\mathcal{A}$ asks the hash value of $a b P$ during the simulation. As done in several papers in the literature (see [8] or [10]), as long as the simulation of the attack's environment is perfect, the probability for $\mathrm{AskH}_{2}$ to happen is the same as in a real attack (i.e. an attack where $\mathcal{A}$ interacts with real oracles). In a real attack we have

$$
\operatorname{Pr}\left[b=b^{\prime}\right] \leq \operatorname{Pr}\left[b=b^{\prime} \mid \neg \mathrm{AskH}_{2}\right] \operatorname{Pr}\left[\neg \mathrm{AskH}_{2}\right]+\operatorname{Pr}\left[\mathrm{AskH}_{2}\right]=\frac{1}{2}+\frac{1}{2} \operatorname{Pr}\left[\mathrm{AskH}_{2}\right]
$$

and then we have $\epsilon=2 \operatorname{Pr}\left[b=b^{\prime}\right]-1 \leq \operatorname{Pr}\left[\mathrm{AskH}_{2}\right]$. Now, the probability that the simulation is not perfect remains to be assessed. The only case where it can happen is when a valid ciphertext is rejected in a de-signcryption query. It is easy to see that for every pair $\left(V_{i}, h_{3, i}\right)$ in $L_{3}$, there is exactly one pair $\left(h_{1, i}, h_{2, i}\right)$ of elements in the range of oracles $H_{1}$ and $H_{2}$ providing a valid ciphertext. The probability to reject a valid ciphertext is thus not greater than $q_{H_{3}} / 2^{2 k}$. The bound on $\mathcal{B}$ 's computation time derives from the fact that every de-signcryption query requires at most 4 pairing evaluations while the extraction of the solution from $L_{2}$ implies to compute at most $2 q_{H_{2}}$ pairings.

The above security proof makes use of the pairing's bilinearity to handle designcryption queries and thus avoids the use of constructions such as [2], 23], 13], [12] that would increase the ciphertext's length or imply additional computation in the de-signcryption operation (this is one of the interests in working with Gap Diffie-Hellman groups). This results in a worst-case bound on algorithm $\mathcal{B}$ 's computation time that seems to be loose: to extract the solution of the $\mathrm{CDH}$ problem, $\mathcal{B}$ might have to compute up to $2 q_{H_{2}}$ pairings if $\mathcal{A}$ only queries oracle $H_{2}$ on tuples of the form $(a P, b P,$.$) . If we allow up to 2^{60} H_{2}$-queries, this appears to be a loose bound at first sight. But we stress that, heuristically, if $\mathcal{A}$ asks many hash queries of tuples $(a P, b P,$.$) that are not valid Diffie-Hellman tuples,$ that means it has no better strategy to find information about the challenge ciphertext than computing the XOR of the ciphertext's $W$-component with hash values of random tuples. Such a strategy would not be more efficient for $\mathcal{A}$ than an exhaustive search of the solution to the Diffie-Hellman instance embedded in 
the challenge ciphertext. An attacker having a non-negligible advantage against the semantic security would ask much less than $2^{60}$ hash queries of invalid DiffieHellman tuple. We can thus expect that, at the end of the simulation, $L_{2}$ only contains a limited number of entries $(a P, b P,$.$) .$

We note that the bound on $\mathcal{B}$ 's probability of success is tight: if we allow $q_{D S C} \leq 2^{30}$ and $q_{H_{3}} \leq 2^{60}$, with $k \geq 160$, we obtain $q_{H_{3}} q_{D S C} / 2^{2 k} \leq 2^{-230}$ which is a negligible function of the parameter $k$.

The following theorem claims the strong unforgeability of the scheme.

Theorem 2. In the random oracle model, if there exists an adversary $\mathcal{F}$ that has a non-negligible advantage $\epsilon$ against the SC-SUF-CMA security of the scheme when running in a time $t$, making $q_{S C}$ signcryption queries, $q_{D S C}$ de-signcryption queries and at most $q_{H_{i}}$ queries on oracles $H_{i}($ for $i=1, \ldots, 4)$, then there exists an algorithm $\mathcal{B}$ that can solve the Diffie-Hellman problem in $\mathbb{G}_{1}$ with a probability $\epsilon^{\prime}>\epsilon-q_{S C} q_{H_{1}} / 2^{k}-q_{D S C} q_{H_{3}} / 2^{2 k}$ in a time $t^{\prime}<t+4 q_{D S C} t_{e}$ where $t_{e}$ denotes the time required for a pairing evaluation.

Proof. given in the full paper ([19]).

This time, we obtain bounds that are explicitly tight. With $k \geq 160$, if we allow $q_{H_{3}}<2^{60}$ and $q_{H_{1}}<2^{50}, q_{D S C}<2^{30}$ we have $q_{S C} q_{H_{1}} / 2^{k}<1 / 2^{80}$ and we still have $q_{D S C} q_{H_{3}}<2^{-230}$. We thus have a negligible degradation of $\mathcal{B}$ 's probability of success when compared to the adversary's advantage. The bound on $\mathcal{B}$ 's running time is also reasonably tight for $q_{D S C}<2^{30}$.

The theorem below claims the ciphertext anonymity property of the scheme.

Theorem 3. In the random oracle model, assume there exists a PPT distinguisher $\mathcal{D}$ that has a non-negligible advantage against the SC-INDK-CCA security of the scheme when running in a time $t$, performing $q_{S C}$ signcryption queries, $q_{D S C}$ de-signcryption queries and $q_{H_{i}}$ queries to oracle $H_{i}$ (for $i=$ $1, \ldots, 4)$. Then there exists an algorithm $\mathcal{B}$ that solves the $C D H$ problem with an advantage $\epsilon^{\prime}>\epsilon-1 / 2^{n+\ell-1}-q_{D S C} q_{H_{3}} / 2^{2 k}$ when running in a time $t^{\prime}<$ $t+\left(4 q_{D S C}+2 q_{H_{2}}\right) t_{e}$ where $t_{e}$ denotes the time required for one pairing evaluation.

Proof. given in the full paper ([19]).

Again, the bound on $\mathcal{B}$ 's computation time might seem to be meaningless but, as for the proof of theorem 1, we can argue that a distinguisher performing many $\mathrm{H}_{2}$ queries on invalid Diffie-Hellman tuples would have no better strategy than an exhaustive search for Diffie-Hellman instances embedded in the challengeciphertext. However, if we look at the proofs of semantic security and ciphertext anonymity for the scheme described in [10], although no bound is explicitly given for the running time of solvers for the bilinear Diffie-Hellman problems, these bounds are not tighter than ours. Furthermore, the proof of ciphertext anonymity provided in [10] leads to a significant degradation of the solver's advantage when compared to the distinguisher's one.

We close this section with the following theorem related to the key invisibility. 
Theorem 4. In the random oracle model, if there exists a distinguisher $\mathcal{D}$ having a non-negligible advantage $\epsilon$ against the SC-INVK-CCA security of the scheme when running in a time $t$ and performing $q_{H_{i}}$ queries to oracles $H_{i}$, for $i=$ $1, \ldots, 4, q_{S C}$ signcryption queries and $q_{D S C}$ de-signcryption queries, then there exists an algorithm $\mathcal{B}$ that solves the $C D H$ problem with an advantage $\epsilon^{\prime}>$ $\epsilon-1 / 2^{n+\ell-1}-q_{D S C} q_{H_{3}} / 2^{2 k}$ in a time $t^{\prime}<t+\left(4 q_{D S C}+2 q_{H_{2}}\right) t_{e}$ where $t_{e}$ is the time required for a pairing evaluation.

Proof. given in the full paper ([19]).

\section{Conclusions}

We proposed a new Diffie-Hellman based signcryption scheme satisfying strong security requirements. It turns out to be the discrete log based signcryption protocol whose unforgeability is the most tightly related to the Diffie-Hellman problem (except the construction in [17, all provably secure solutions are built on signatures having a security proof relying on the forking lemma (24],25]) and the CCA-security of [17 relies on stronger assumptions than the present scheme). By heuristic arguments, we argued that the reduction from an adaptive chosen ciphertext adversary to a solver for the Diffie-Hellman problem is also efficient. We also introduced a security notion called 'key invisibility' that can be shown to imply 'key privacy' in some cases (see [19] for details).

\section{References}

1. J.-H. An, Y. Dodis and T. Rabin. On the Security of Joint Signature and Encryption. In Advances in Cryptology - Eurocrypt'02, LNCS 2332, pp. 83-107. Springer, 2002.

2. J. Baek, B. Lee and K. Kim. Secure Length-Saving ElGamal Encryption under the Computational Diffie-Hellman Assumption. In Proceedings of ACISP'00, LNCS 1841, pp. 49-58. Springer, 2000.

3. J. Baek, R. Steinfeld and Y. Zheng. Formal Proofs for the Security of Signcryption. In Proceedings of PKC'02,LNCS 2274, pp. 80-98. Springer, 2002.

4. J. Baek and Y. Zheng. Simple and Efficient Threshold Cryptosystem from the Gap Diffie-Hellman Group. Available at http://citeseer.nj.nec.com/567030.html.

5. F. Bao and R.-H. Deng. A Signcryption Scheme with Signature Directly Verifiable by Public Key. In Proceedings of PKC'98, LNCS 1998, pp. 55-59, 1998.

6. M. Bellare, A. Boldyreva, A. Desai and D. Pointcheval. Key-Privacy in Public-Key Encryption. In Advances in Cryptology - Asiacrypt'01, LNCS 2248, pp. 566-582. Springer, 2001.

7. M. Bellare, P. Rogaway, Random oracles are practical: A paradigm for designing efficient protocols, Proc. of the $1^{\text {st }} \mathrm{ACM}$ Conference on Computer and Communications Security, pp. 62-73, 1993.

8. D. Boneh and M. Franklin. Identity Based Encryption From the Weil Pairing. In Advances in Cryptology - Proceedings of Crypto'01, LNCS 2139, pp. 213-229. Springer, 2001.

9. D. Boneh, B. Lynn and H. Shacham. Short Signatures from the Weil Pairing. In Advances in Cryptology - Proceedings of Asiacrypt'01, LNCS 2248, pp. 514-532. Springer, 2001. 
10. X. Boyen. Multipurpose Identity-Based Signcryption: A Swiss Army Knife for Identity-Based Cryptography. In Advances in Cryptology - Crypto'03, LNCS 2729, pp. 382-398. Springer, 2003.

11. Y. Dodis, M.-J. Freedman and S. Walfish. Parallel Signcryption with OAEP, PSSR and other Feistel Paddings. 2003. Available at http://eprint.iacr.org/2003/043/.

12. E. Fujisaki and T. Okamoto. Secure Integration of Asymmetric and Symmetric Encryption Schemes. In Advances in Cryptology - Crypto'99, LNCS 1666, pp. 537-554. Springer, 1999.

13. E. Fujisaki and T. Okamoto. How to Enhance the Security of Public-Key Encryption at Minimum Cost. In Proceedings of PKC'99, LNCS 1560, pp. 53-68. Springer, 1999.

14. S. Galbraith and W. Mao. Invisibility and Anonymity of Undeniable and Confirmer Signatures. In Topics in Cryptology - CT-RSA 2003, LNCS 2612, pp. 80-97. Springer, 2003.

15. C. Gamage, J. Leiwo and Y. Zheng. Encrypted Message Authentication by Firewalls. In Proceedings of PKC'98, LNCS 1560, pp. 69-81. Springer, 1998.

16. E.-J. Goh and S. Jarecki. A Signature Scheme as Secure as the Diffie-Hellman Problem. In Advances in Cryptology - Eurocrypt'03, LNCS 2656, pp. 401-415. Springer, 2003.

17. I.-R. Jeong, H.-Y. Jeong, H.-S. Rhee, D.-H. Lee and I.-L. Jong. Provably Secure Encrypt-then-Sign Composition in Hybrid Signcryption. In Proceedings of ICISC'02, LNCS 2587, pp. 16-34. Springer, 2002.

18. A. Joux and K. Nguyen. Separating Decision Diffie-Hellman from Diffie-Hellman in Cryptographic Groups. In Journal of Cryptology, vol. 16-Number 4, pp. 239-247. Springer, 2003.

19. B. Libert and J.-J. Quisquater, Efficient Signcryption with Key Privacy from Gap Diffie-Hellman Groups. Full paper, available on http://eprint.iacr.org.

20. J. Malone-Lee and W. Mao. Two Birds One Stone: Signcryption using RSA. In Topics in Cryptology - CT-RSA 2003, LNCS 2612, pp. 211-225. Springer, 2003.

21. T. Okamoto and D. Pointcheval. The Gap-Problems: A New Class of Problems for the Security of Cryptographic Schemes. In Proceedings of PKC'01, LNCS 1992. Springer, 2001.

22. J. Pieprzyk and D. Pointcheval. Parallel Authentication and Public-Key Encryption. In Proceedings of ACISP'03, LNCS 2727, pp. 383-401. Springer, 2003.

23. D. Pointcheval. Chosen-Ciphertext Security for any One-Way Cryptosystem . In Proceedings of PKC'00, LNCS 1751, pp. 129-146. Springer, 2000.

24. D. Pointcheval and J. Stern. Security Proofs for Signature Schemes. In Advances in Cryptology - Eurocrypt'96, LNCS 1992, pp. 387-398. Springer, 1996.

25. D. Pointcheval and J. Stern. Security Arguments for Digital Signatures and Blind Signatures. In Journal of Cryptology, vol. 13-Number 3, pp. 361-396. Springer, 2000 .

26. J.-B. Shin, K. Lee and K. Shim. New DSA-verifiable Signcryption Schemes. In Proceedings of ICISC'02, LNCS 2587, pp. 35-47. Springer, 2002.

27. R. Steinfeld and Y. Zheng. A Signcryption Scheme Based on Integer Factorization. In Proceedings of ISW'00, LNCS 1975, pp. 308-322, Springer, 2000.

28. B.-H. Yum and P.-J. Lee. New Signcryption Schemes Based on KCDSA. In Proceedings of ICISC'01, LNCS 2288, pp. 305-317, Springer, 2001.

29. Y. Zheng. Digital signcryption or how to achieve cost (signature \& encryption) $<<\operatorname{cost}($ signature) $+\operatorname{cost}($ encryption). In Advances in Cryptology - Crypto'97, LNCS 1294, pp. 165-179, Springer, 1997. 